\title{
Automatic delineation and quantification of pulmonary vascular obstruction index in patients with pulmonary embolism using Perfusion SPECT-CT: a simulation study
}

David Bourhis ${ }^{1,2^{*}}$, Laura Wagner ${ }^{1}$, Julien Rioult ${ }^{1}$, Philippe Robin ${ }^{1,2}$, Romain Le Pennec ${ }^{1,2}$, Cécile Tromeur ${ }^{2,3}$, Pierre Yves Salaün ${ }^{1,2}$ and Pierre Yves Le Roux ${ }^{1,2}$

\author{
*Correspondence: david.bourhis@ \\ chu-brest.fr \\ 'Service de Médecine Nucléaire, \\ Centre Hospitalier Régional \\ Universitaire de Brest, Brest, France \\ ${ }^{2}$ EA3878 GETBO, Université de \\ Bretagne Occidentale, Brest, France \\ Full list of author information is \\ available at the end of the article
}

\begin{abstract}
Background: In patients with pulmonary embolism (PE), there is a growing interest in quantifying the pulmonary vascular obtruction index (PVOI), which may be an independent risk factor for PE recurrence. Perfusion SPECT/CT is a very attractive tool to provide an accurate quantification of the PVOI. However, there is currently no reliable method to automatically delineate and quantify it. The aim of this phantom study was to assess and compare 3 segmentation methods for PVOI quantification with perfusion SPECT/CT imaging.
\end{abstract}

Methods: Three hundred ninety-six SPECT/CT scans, with various PE scenarios $(n=44)$, anterior to posterior perfusion gradients $(n=3)$, and lung volumes $(n=3)$ were simulated using Simind software. Three segmentation methods were assesssed: (1) using an intensity threshold expressed as a percentage of the maximal voxel value (MaxTh), (2) using a Z-score threshold (ZTh) after building a Z-score parametric lung map, and (3) using a relative difference threshold (RelDiffTh) after building a relative difference parametric map. Ninety randomly selected simulations were used to define the optimal threshold, and 306 simulations were used for the complete analysis. Spacial correlation between PE volumes from the phantom data and the delineated PE volumes was assessed by computing DICE $E_{P E}$ indices. BlandAltman statistics were used to calculate agreement for PVOI between the phantom data and the segmentation methods.

Results: Mean DICE compared with the MaxTh method $(0.78 \pm 0.16)$ and the ZTh method $(0.67 \pm 0.15)$. Using the RelDiffTh method, mean DICE the perfusion gradient and the lung volumes. Using the RelDiffTh method, mean relative difference in PVOI was - 12\%, and the limits of agreement were - $40 \%$ to $16 \%$. Values were $3 \%$ (- $75 \%$ to $81 \%$ ) for MaxTh method and $0 \%$ (- 120\% to 120\%) for ZTh method. Graphycal analysis of the Bland-Altman graph for the RelDiffTh method showed very close estimation of the PVOI for small and medium PE, and a trend toward an underestimation of large PE. 
Conclusion: In this phantom study, a delineation method based on a relative difference parametric map provided a good estimation of the PVOI, regardless of the extent of PE, the intensity of the anterior to posterior gradient, and the whole lung volumes.

Keywords: V/Q SPECT-CT, Lung function, PE, Simulation, Segmentation

\section{Background}

Lung ventilation/perfusion (V/Q) scintigraphy is a well established test for pulmonary embolism (PE) diagnosis [1, 2]. V/Q planar scan has been validated in large management oucome studies [3-5]. Development of imaging equipment and radiopharmaceuticals has then allowed the introduction of V/Q single photon emission computed tomography (SPECT) scintigraphy, and more recently V/Q SPECT/CT, which has been reported to improve the diagnostic performance of the test $[6,7]$ and has been widely adopted in daily practice [8]. Besides the diagnosis of PE itself, there has been a growing interest in quantifying the extent of vascular obstruction of PE. Indeed, several studies reported that the pulmonary vascular obstruction index (PVOI) measured on lung perfusion scintigraphy, either at diagnosis or at completion of anticoagulant therapy, may be an independent risk factor for PE recurrence and chronic thromboebolic pulmonary hypertension (CTEPH) $[9,10]$. PVOI quantification by planar imaging relies on the application of the Meyer score [11], which was validated on perfusion imaging using pulmonary angiography as a reference standard, but which is complex to use in daily practice and involves a certain amount of subjectivity. By using 3-dimensional imaging, lung SPECT has an inherent technical advantage over conventional 2dimensional planar imaging through its ability to eliminate overlap of activities, its visualization of the medial-basal segment, and its ability to better characterize the size, shape, and location of defects [12]. Perfusion SPECT/CT is therefore a very attractive tool to improve the accuracy and reproducibility of PVOI quantification. However, there is currently no reliable method to delineate and quantify the PVOI with Perfusion SPECT/CT imaging. Indeed, the delineation of lung perfusion is complex because of the physiological non-uniformity of lung perfusion, with especially an anterior-toposterior intensity gradient of variable intensity across patients. In that respect, similarly to a method used for brain imaging (Statistical Parametric Mapping (SPM) [13]), a method based on the co-registration with physiological images and a voxelwize analysis may be of value. Another issue for the assessment and validation of segmentation methods of lung perfusion with perfusion SPECT/CT is the lack of ground truth. In order to overcome these problems, Monte-Carlo simulations may be an intersting tool. First, it would solve the problem of the reference standard. Second, it would allow to test a large number of segmentation methods and clinical scerarios. Finally, simulations may integrate confounding factors, such as the impact of gravity on the macroaggregated albumin distribution, that make conventional delineation methods inacurate in some situations. Our group recently generated 3D mean and standard deviation statistical maps of regional lung function based on free-form co-registration of 73 normal V/ Q SPECT/CT scans, with the aim of testing original methods for lung function delineation based on an a-priori knowledge of normality [14]. In this work, we found that the main factor of variability across patients with a normal perfusion SPECT/CT was the 
intensity of the anterior to posterior gradient. In a second work, we therefore developed and validated a dual isotopes lung V/Q SPECT-CT model for Monte Carlo studies, integrating the anterior-to-posterior gradient on perfusion images [15].

The aim of this phantom study was to test and compare 3 different delineation methods to automatically compute the PVOI on perfusion SPECT/CT imaging: a conventional method using the same intensity threshold for all pixels, and 2 original methods based on the coregistration and comparison with physiological statistical maps, and a voxelwise analysis (Z-score threshold and relative difference threshold methods).

\section{Materials and methods}

\section{Overall methology for Perfusion SPECT/CT simulations}

In order to test the various delineation methods, we simulated a large spectrum of PE, with various size and location (44 clinical scenarios), on lung perfusion SPECT/CT. Three different sizes of lungs were simulated in order to assess the impact of the statistical maps' deformable registrations. As the variability of the intensity of the anterior to posterior gradient is a key issue for the delineation of lung perfusion volumes, we also simulated three different anterior to posterior intensity gradients. Overall, $44 \times 3 \times 3=$ 396 perfusion SPECT/CT scans were therefore simulated. As PVOI is a quantification of lung perfusion, ventilation delineation was not assessed in this work, but it was integrated in the model to improve the realism of simulations in terms of Compton scattering.

\section{PE models definition}

To define a catalog of PE, CT examinations of real patients were delineated. A senior nuclear medicine physician with experience in reading V/Q scan delineated the lobes following the fissures and the lung segments based on the bronchial tree. The segments were then divided to obtain two half segments. We defined 44 models of PE, selecting the lobes, segments, and sub-segments to be turned off for simulations. We defined 8 sub-segmental PE (one or multiple sub-segments), 10 single segmental PE, 19 multi segmental PE (from 2 to 14 segments), 5 lobar PE, and 2 multi lobar PE. The exact definition of the models can be found in the supplementary material.

\section{Lung SPECT simulations and reconstructions}

All simulations were run with Simind software [16]. Realistic dual isotopes lung V/Q SPECT scans were simulated using a methodology described in a previous work [15]. Briefly, we used CT data from real SPECT-CT examinations acquired on a Symbia T6 system (Siemens, Erlangen) equipped with a medium energy low penetration (MELP) collimator. The camera modeling parameters were set in order to correspond to this system as it is used for dual isotopes V/Q SPECT-CT [17]. CT data (low-dose free breathing CT) were used to define three simulation digital phantoms corresponding to small $(1915 \mathrm{~mL})$, regular $(2730 \mathrm{~mL})$, and large $(3515 \mathrm{~mL})$ lungs. These scans were selected so that the lung volume fit the mean, the mean plus one, and mean minus one standard deviation volume, measured on the database of 73 normal cases [14]. Simulation geometries were Zubal-like phantoms $[18,19]$, built from the CT. CT data were 
segmented according to hounsfield units using MiM software (7.0, Cleveland). Six representative tissues were delineated, including outside air, bones, fat, soft tissues, lungs, bronchi, and the PE area. A code was assigned to each area. Images bit depth was set to 8 bits, and a unique value was attributed to each segmented area using ImageJ sofware [20]. Those values were used in Simind to set the desired value of density and radioactivity concentration in the defined areas. Digital phantom was subsampled in a $128^{2}$ matrix to accelerate the simulation calculation, and the simulation grid was $128 \times 128 \times 108$ matrix, corresponding to $3.92 \times 3.92 \times 3.59 \mathrm{~mm}$ voxels. With regard to ventilation, the simulated radioactivity was evenly set to $55 \mathrm{kBq} \cdot \mathrm{mL}^{-1}$ in the lungs and the airways. With regard to perfusion, in order to model the anterior to posterior gradient, lungs were divided into sixteen coronal planes. For each coronal plane, a relative to maximum radioactivity concentration value was assigned. Three different sets of values were defined to model a weak, regular, and a strong gradient. The gradients were defined to fit the mean, the mean plus one, and mean minus one standard deviation anterior to posterior intensity gradient measured on the database of 73 normal cases [14]. Radioactivity concentrations rose from the first to the last coronal plane to define weak, regular, and strong gradients (64 to $85 \mathrm{kB} \cdot \mathrm{mL}^{-1}, 49$ to $98 \mathrm{kB} \cdot \mathrm{mL}^{-1}$ and 29 to $116 \mathrm{kB} \cdot \mathrm{mL}^{-1}$ ) for the small phantom, 52 to $67 \mathrm{kB} \cdot \mathrm{mL}^{-1}, 42$ to $78 \mathrm{kB} \cdot \mathrm{mL}^{-1}$ and 27 to $95 \mathrm{kB} \cdot \mathrm{mL}^{-1}$ for the regular phantom, and 44 to $56 \mathrm{kB} \cdot \mathrm{mL}^{-1}, 36$ to $67 \mathrm{kB} \cdot \mathrm{mL}^{-1}$, and 24 to $85 \mathrm{kB} . \mathrm{mL}^{-1}$ for the large phantom. As the source map is different when simulating ventilation and perfusion, simulations were not run simultaneously. Photons emitted from ${ }^{99 \mathrm{~m}}$ Tc decay were simulated with a $140 \mathrm{keV}$ energy and $88.5 \%$ abundance. As Krypton gas is continuously inspired and expired and has a very fast decay (half-life is $13 \mathrm{~s}$ ), it was simulated as a stationary gas without significant decay, with homogeneous concentration, with a $190 \mathrm{keV}$ energy and $100 \%$ abundance. Scatter data was stored at each energy window, and ${ }^{81 \mathrm{~m}} \mathrm{Kr}$ scatter was added to ${ }^{99 \mathrm{~m}} \mathrm{Tc}$ lower scatter and primary energy windows. All SPECT reconstructions were performed on Siemens MIApps software , with FLASH3D, 4 iterations, 8 subsets, and $8.4 \mathrm{~mm}$ gaussian post filtering, scatter correction (double energy windows method), with attenuation correction. Overall, we defined 44 models of PE, simulated on three different geometries (small, regular, and large lungs) with three different radioactivity source maps (weak, regular, and strong anterior to posterior intensity gradient). Three hundred ninety-six datasets were therefore reconstructed.

\section{Segmentation methods}

We evaluated three segmentation methods of lung volumes with normal perfusion. First, we tested a fixed intensity threshold for all pixels, expressed as a percentage of the maximal pixel value (MaxTh), with six different thresholds (10\%, 15\%, 20\%, 25\%, $30 \%, 35 \%)$, applied on both lungs. Then, we tested two original methods, based on the quantification of abnormality, inspired by the SPM method used for brain imaging: a Z-score map threshold method (ZTh), and a relative difference map threshold (RelDiffTh) method. These methods were applied using mean (NMmap) and a standard deviation (NSDmap) maps built in a previous study from 73 normal V/Q SPECT/CT cases [14]. For each simulation dataset, we performed a free-form registration, based on CT data, of the NMmap and NSDmap up to the simulated SPECT-CT. Then, we 
normalized the simulated SPECT to the mean intenity value, and we computed two parametric maps, a Z-score map (Zmap) and a relative difference map (RelDiffmap) as follows:

$$
\begin{aligned}
& \operatorname{Zmap}_{\text {simulated SPECT }}(x, y, z)=\frac{[\text { normSPECTPixelvalue }(x, y, z)-\operatorname{NMmapValue}(x, y, z)]}{\operatorname{NSDmapValue}(x, y, z)} ; \\
& \operatorname{RelDiffmap}_{\text {simulated SPECT }}(x, y, z)=\frac{[\text { normSPECTPixelvalue }(x, y, z)-\operatorname{NMmapValue}(x, y, z)]}{\operatorname{NMmapValue}(x, y, z)} ;
\end{aligned}
$$

An example of the different datasets of one case is shown in Fig. 1. Finally, we tested several thresholds on Zmap (- 0.6, - 0.8, - 1, - 1.2, - 1.4, - 1.6) and RelDiffmap (- 30\%, - 40\%, - 50\%, - 60\%).

As the threshold tool delineated pixels whose intensities were higher than the threshold values, only the functional lung volumes where segmented for the three methods.

\section{Quantitative measurements}

The whole lung volume (WLv) was the volume computed from CT data. From the phantom data, the ground truth volumes were computed, including the functional

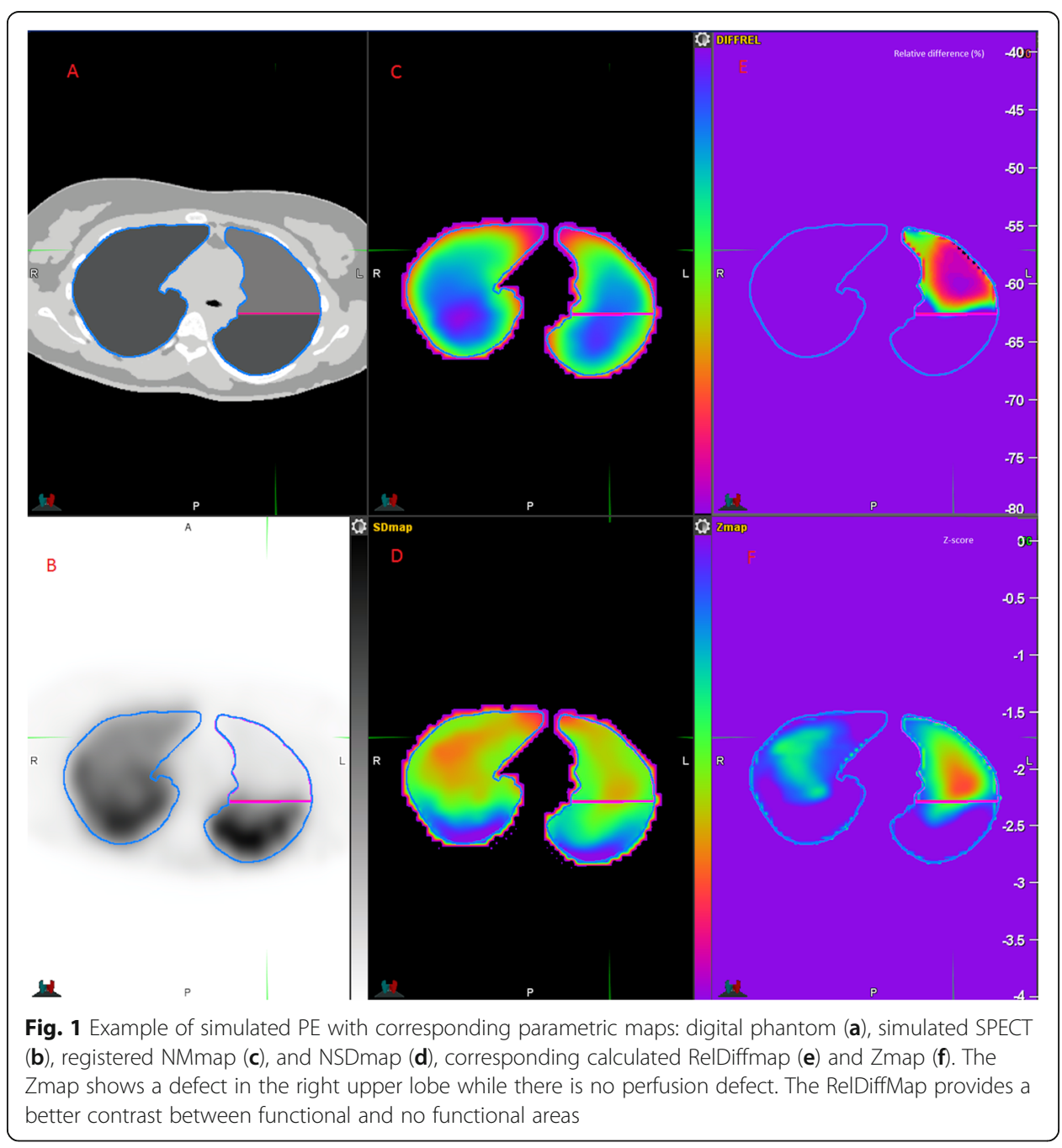


lungs volume $\left(\mathrm{FLv}_{\mathrm{ph}}\right)$ and the PE volume $\left(\mathrm{PEv}_{\mathrm{ph}}\right)$. Ground truth PVOI was calculated as follows : $\mathrm{PVOI}_{\mathrm{ph}}=\mathrm{PEv}_{\mathrm{ph}} / \mathrm{WLv}$. For each simulation, segmentation method and threshold value, the segmented functional lungs $\left(\mathrm{FLv}_{\mathrm{s}}\right)$ were stored and the PE volume $\left(\mathrm{PEv}_{\mathrm{s}}\right)$ and simulation PVOI were calculated as follows: $\mathrm{PEv}_{\mathrm{s}}=\mathrm{WLv}-\mathrm{FLv}_{\mathrm{s}}, \mathrm{PVOI}_{\mathrm{s}}=$ $\mathrm{PEv}_{\mathrm{s}} / \mathrm{WLv}$. To measure the efficiency of the segmentation, DICE indices (DICE $\mathrm{PE}$ ) of segmented $\mathrm{PE}$ volumes $\left(\mathrm{PEv}_{\mathrm{s}}\right)$ and phantom $\mathrm{PE}$ volumes $\left(\mathrm{PEv}_{\mathrm{ph}}\right)$ were calculated as follows DICE $E_{\mathrm{PE}}=\left(2 \times \mathrm{PEv}_{\mathrm{s}} \cap \mathrm{PEv}_{\mathrm{ph}}\right) /\left(\mathrm{PEv}_{\mathrm{s}}+\mathrm{PEv}_{\mathrm{ph}}\right)$.

\section{Statistical analysis}

All measurements were separated in two batches. 10 models of PE (90 simulations) were randomly selected to define the best threshold for each segmentation method. The best threshold was defined as the threshod which provided the highest mean DICE $_{\mathrm{PE}}$ index for each method. Thirty-four models (306 simulations) were available for the complete analysis. DICE $\mathrm{PE}_{\mathrm{PE}}$ were expressed graphically as [min;Q1;median;mean;Q3; $\max ]$ and quantitatively as mean $( \pm \mathrm{SD})$. DICE PE indexes distributions were compared using a Wilcoxon signed rank test. A p value $<0.05$ was considered statistically significant. The correlations between $\mathrm{PVOI}_{\mathrm{ph}}$ and $\mathrm{PVOI}_{\mathrm{s}}$ were tested using Pearson's $\mathrm{r}$ correlation coefficient, and a Bland and Altman visual analysis was performed in terms of absolute difference and relative difference.

\section{Results}

\section{Calibration}

Using the MaxTh method, mean $( \pm \mathrm{SD}) \mathrm{DICE}_{\mathrm{PE}}$ coefficients were $0.59(0.23), 0.66$ (0.18), 0.62 (0.18), 0.55 (0.19), 0.48 (0.19), and $0.2(0.18)$ with the $10 \%, 15 \%, 20 \%, 25 \%$, $30 \%$, and $35 \%$ thresholds, respectiveley. Using the ZTh method, mean $( \pm$ SD) DICE coefficients were $0.53(0.2), 0.56$ (0.18), 0.57 (0.15), 0.56 (0.13), 0.53 (0.13), and 0.47 (0.12) with the $-0.6,-0.8,-1,-1.2,-1.4$, and -1.6 thresholds, respectiveley. Using the RelDiffTh methods, mean $( \pm \mathrm{SD}) \mathrm{DICE}_{\mathrm{PE}}$ coefficients were $0.67(0.2), 0.77(0.14)$, 0.81 (0.11), and $0.75(0.16)$ with the $-30 \%,-40 \%,-50 \%$, and $-60 \%$ thresholds, respectiveley (see Fig. 2).

\section{Segmentation methods evaluation Comparison of methods}

Spatial correlation between $\mathrm{PE}_{\mathrm{s}}$ and $\mathrm{PE}_{\mathrm{ph}}$ volumes, as assessed by the $\mathrm{DICE}_{\mathrm{PE}}$ index, is presented in Fig. 3. Mean DICE $\mathrm{PE}_{\mathrm{PE}}$ index was significantly higher with the RelDiffTh

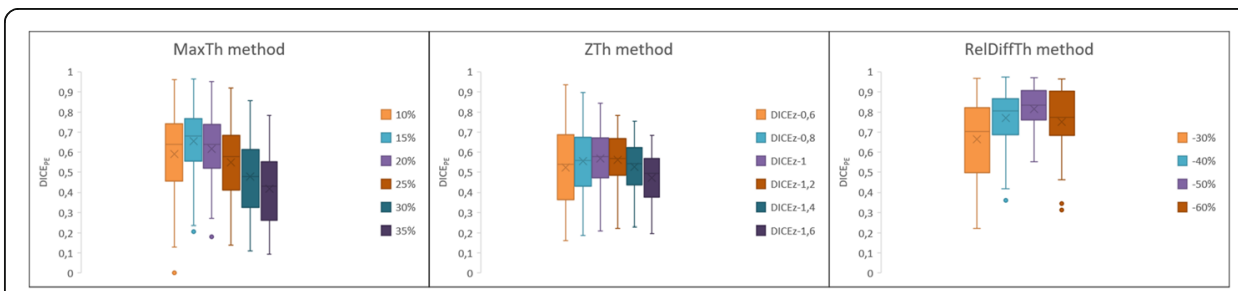

Fig. 2 Determination of the best threshold for the 3 segmentation methods. DICE ${ }_{P E}$ coefficients were calculated on 90 randomly selected models using various thresholds. The best thresholds were $15 \%$ for the MaxTh method, $Z=-1$ for the ZTh method and $-50 \%$ for the RelDiffTh method 


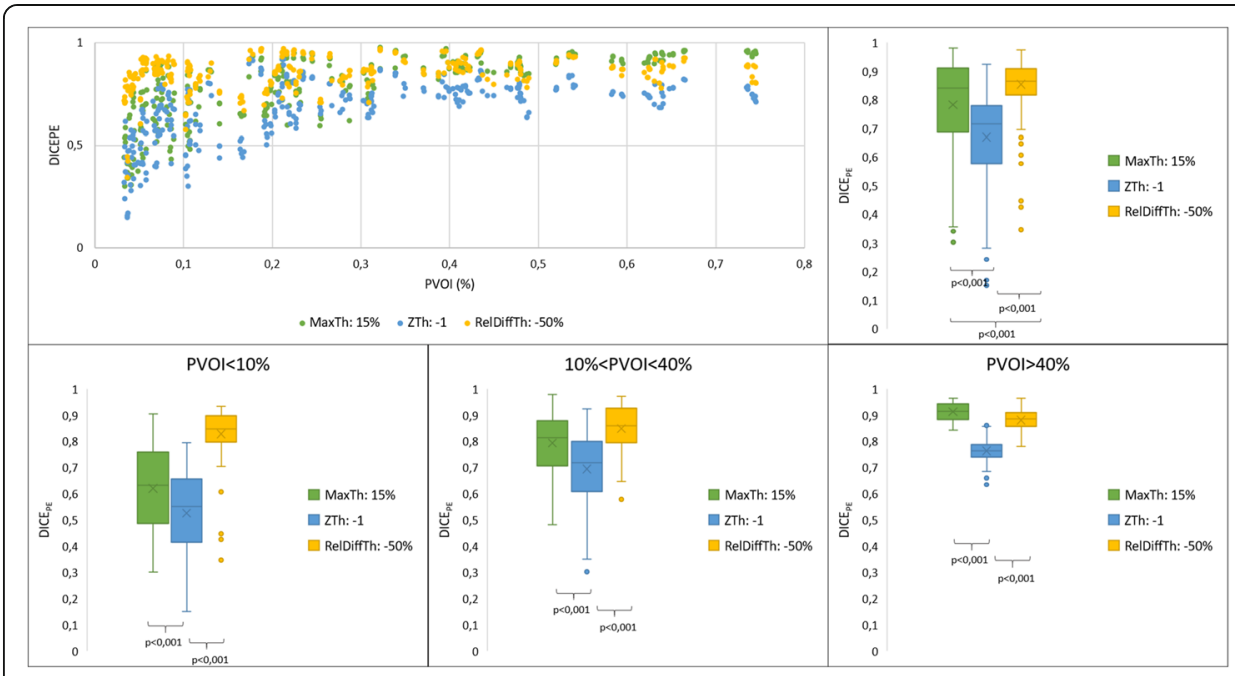

Fig. 3 Comparison of the three segmentation methods in terms of DICEPE indices. DICE $E_{P E}$ are presented for all PE volumes in the first row and according to the PVOI in the second row. RelDiff method with - 50\% threshold shows overall higher DICE $E_{\mathrm{PE}}$ indices, especially for small and medium PE

method $(0.85(0.08))$, as compared with the MaxTh method $(0.78(0.16))$ and the ZTh method $(0.67(0.15))$. Differences between the 3 segmentation methods were more pronouced for small PE (PVOI $<10 \%)$, with a mean DICE $\mathrm{PE}_{\mathrm{PE}}$ coefficient of $0.62(0.17), 0.53$ (0.15), and 0.83 (0.1) using the MaxTh, ZTh, and RelDiffTh method, respectively. For medium sized PVOI $(10 \%<\mathrm{PVOI}<40 \%)$, mean DICE $\mathrm{PE}$ coefficients were $0.79(0.12)$, 0.7 (0.13), and 0.85 (0.09) for MaxTh, ZTh, and RelDiffTh methods, respectively. For large PVOI (PVOI > 40\%), mean DICE $\mathrm{PE}_{\mathrm{PE}}$ coefficients were 0.91 (0.04), $0.76(0.05)$, and 0.88 (0.04) for MaxTh, ZTh, and RelDiffTh methods, respectively.

Figures 4 and 5 showed the impact of the anterior to posterior gradient intensity, lung volume, and PE location on DICE $\mathrm{PE}_{\mathrm{PE}}$ distribution according to the segmentation

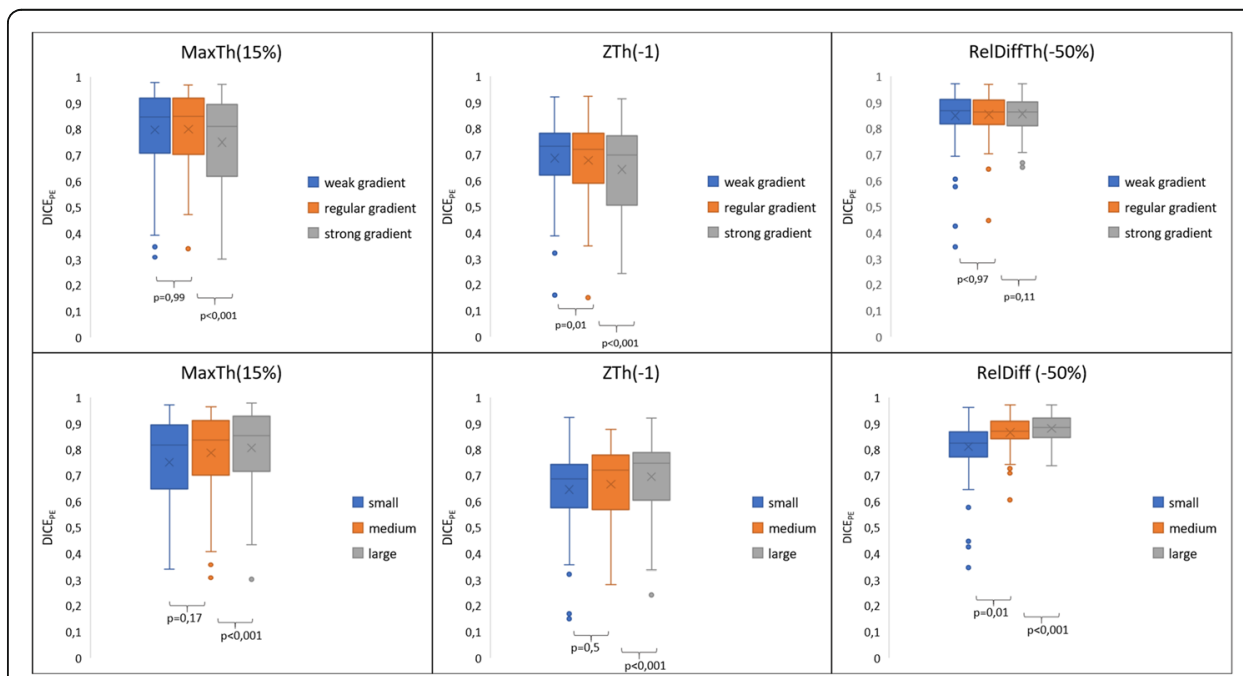

Fig. 4 Influences of intensity gradient strenght (first row) and lung volumes (second row) on DicepE coefficients according to the segmentation methods. Using the RelDiff method with a $-50 \%$ threshold, there was no significant influence of the gradient strength and low influence of lung 


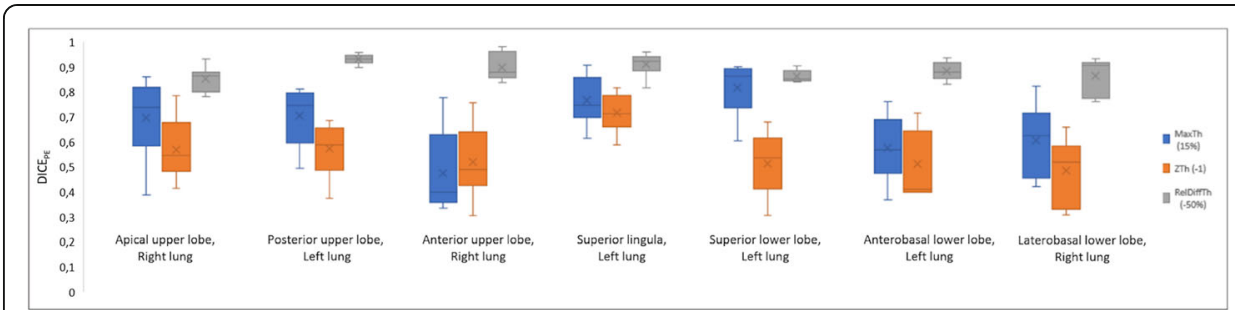

Fig. 5 Influence of PE location: high DICEPE were found using the RelDiff method regardless of the location of $P E$

method. The impact of the gradient was much lower for the ReDiffTh method as compared with the MaxTh and ZTh methods (Fig. 4, first row). Using the ReDiffTh method, mean DICE $\mathrm{PE}_{\mathrm{P}}$ was $0.87(0.08), 0.87(0.06)$, and $0.86(0.06)$ with the weak, regular, and strong gradient respectively. Lung volumes had an impact on the three segmentation methods, but it was more pronounced for the RelDiffTh methods. Using the ReDiffTh method, mean DICE $\mathrm{PE}_{\mathrm{PE}}$ was 0.81 (0.11), $0.87(0.6)$, and $0.81(0.11)$ with the small, medium, and large lung volumes, respectively.

\section{PVOI analysis}

The correlation between PVOIs and PVOIph (Fig. 6) was very high for the three segmentation methods $(r=0.977$ for MaxTh method, $r=0.968$ for ZTh method and $r=$ 0.988 for RelDiffTh method) with a smaller confidence interval for RelDiffTh method. The slope of the regression line was closer to 1 for MaxTh method (0.94 for MaxTh method, $\mathrm{r}=0.55$ for ZTh method and $\mathrm{r}=0.78$ for RelDiffTh method). Bland and Altman analysis is presented in Fig. 7 and Table 1. Mean differences and 95\% limits of agreement are reported in Table 1. Using the MaxTh method, mean absolute difference in PVOI was $-1 \%$, and limits of agreement were -9 to $8 \%$. Mean relative difference in PVOI was $3 \%$, and the limits of agreement were -75 to $81 \%$. Graphical analysis showed that absolute differences were homogenously distributed regardless of the PVOI value, resulting in large relative differences (up to $250 \%$ ) for small PVOI indices $(<10 \%)$. Using the ZTh method, mean absolute difference in PVOI was $-7 \%$, and limits of agreement were - 25 to $12 \%$. Mean relative difference in PVOI was $0 \%$, and the confidence index was -120 to $120 \%$. Graphical analysis showed that PVOI were over-

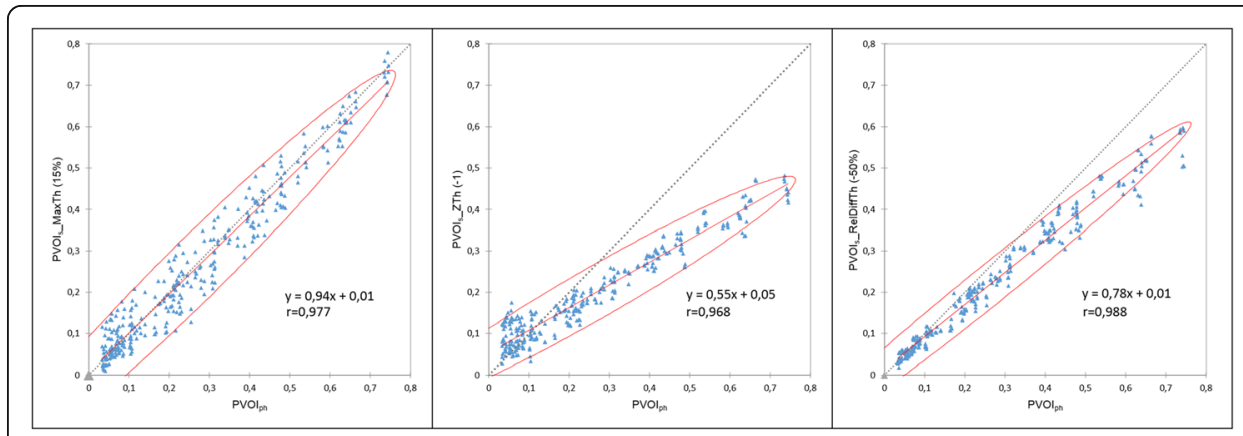

Fig. 6 Pearson's correlation between $\mathrm{PVOI}_{\text {ph }}$ and $\mathrm{PVOI}_{\mathrm{s}}$ for the three segmentation methods. Person's coefficient was higher for RelDiff method, but the slope was closer to 1 for MaxTh method 


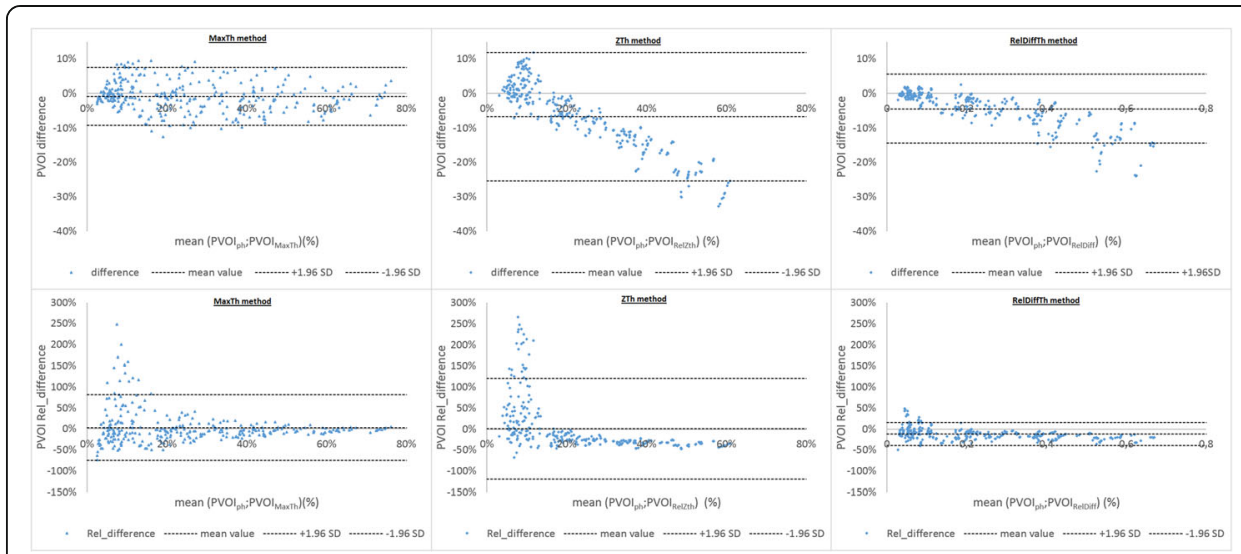

Fig. 7 Bland and Altman analysis of the three segmentation methods in terms of absolute difference (first row) and relative difference (second row). RelDiff method showed lower relative differences for medium and small PE

estimated for small PE and under estimated for large PE. Using the RelDiffTh method, mean absolute difference in PVOI was $-4 \%$, and limits of agreement were -14 to $6 \%$. Mean relative difference in PVOI was $-12 \%$, and the confidence index was - 40 to $16 \%$. Graphical analysis show low absolute and relative errors on small and medium PVOI and a trend toward an underestimation of large PVOI. Examples of delineations of a small PE and a massive PE are shown Figs. 8 and 9.

\section{Discussion}

In this phantom study, we tested and compared three automatic delineation methods of perfusion functional volumes on 396 simulated V/Q SPECT/CT. The best method was a segmentation using a relative difference threshold $(-50 \%)$ after coregistration with a parametric normal mean map, which provided good results in terms of spatial correlation and PVOI measurement, regadless of the extent of PE, the intensity of the anterior to posterior gradient, and the whole lung volumes.

The first delineation method consisted in applying to all pixels a fixed intensity threshold defined as a percentage of the maximal pixel value. This has been the most commonly used method for lung segmentation mainly because of its ease of use [21-24]. The overall results showed relatively good spatial agreement with a mean DICE $\mathrm{PE}=0.78$. Mean relative difference was $-1 \%$ (limits of agreement -75 to $81 \%$ ). However, while spatial correlation was high for large $\mathrm{PE}\left(\mathrm{DICE}_{\mathrm{PE}}=0.91\right.$ for $\left.\mathrm{PVOI}_{\mathrm{PH}}>40 \%\right)$, it was lower for small $\mathrm{PE}\left(\mathrm{DICE}_{\mathrm{PE}}=0.62\right.$ for $\left.\mathrm{PE}<10 \%\right)$, showing that the method is inaccurate to delineate small PE. Of note is that there was a good correlation in PVOI estimation, although the spatial agreement was poor (low DICE $_{\mathrm{PE}}$ value), because segmented non PE

Table 1 Bland and Altman analysis: mean absolute and relative differences, with limits of agreement for the three segmentation methods

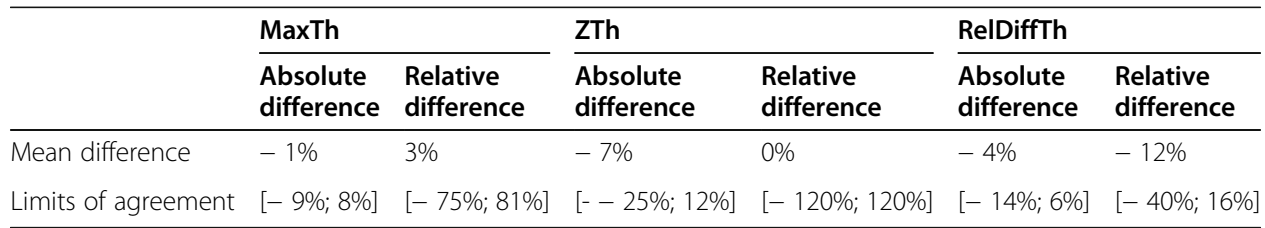



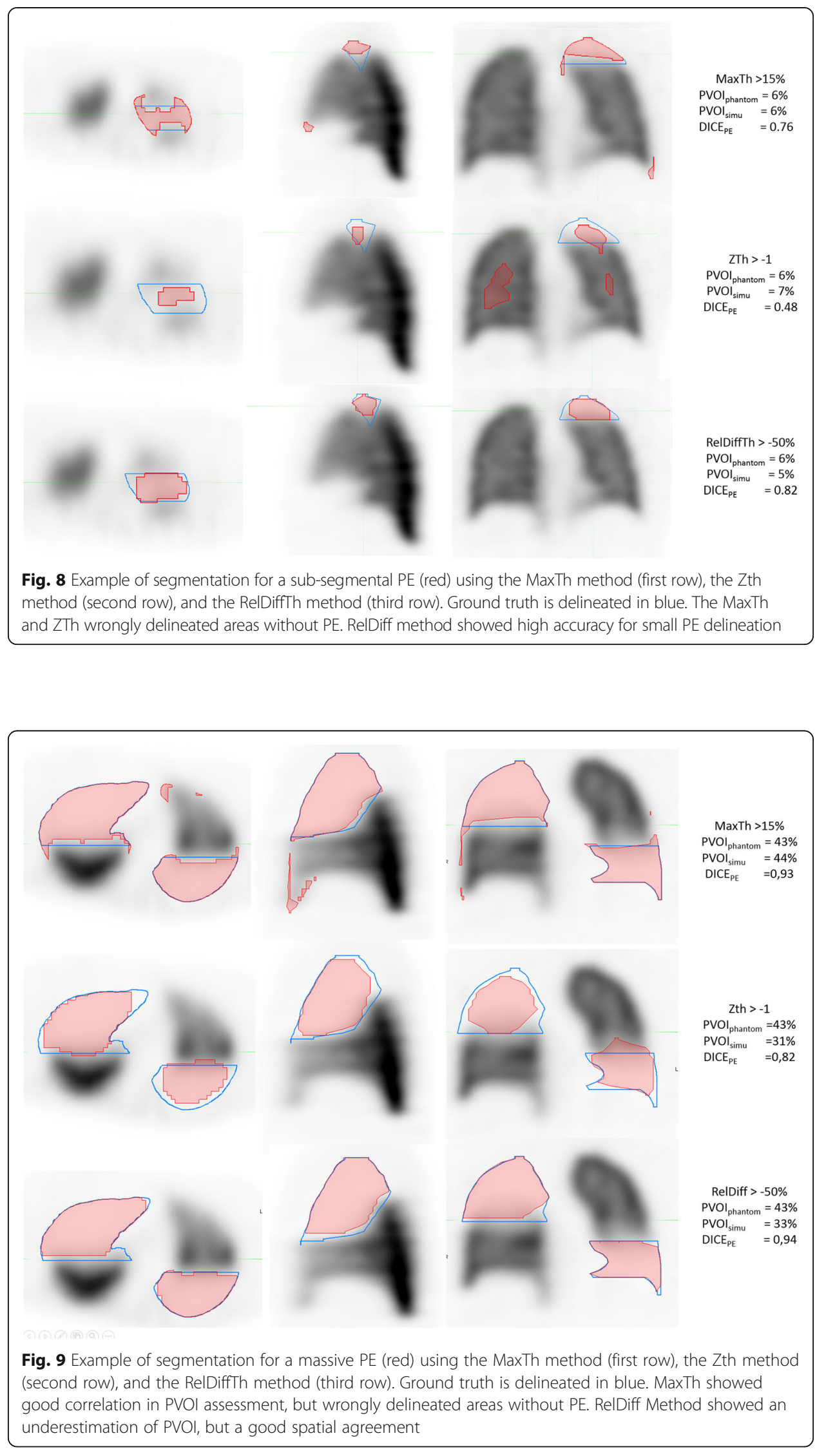
volumes were close to non segmented PE volumes (see Fig. 8). Bland and Altman analysis showed a large overestimation of PVOI in a significant proportion of patients with small PE. As illustrated in Fig. 8, this overestimation was mainly due to the delineation of non $\mathrm{PE}$ areas around the lungs, especially in their anterior aspect. In a clinical perspective, this represents a major limitation of the method. Indeed, several studies reporting on the pronostic value of PVOI at completion of anticoagulation therapy to predict the risk of PE recurrence or CTEPH, used a PVOI cut-off of $5 \%$ or 10\% [9]. Accordingly, an overestimation of the PVOI of small PE (e.g., 15-20\% while the actual size is $<10 \%$ ) may have an impact on the therapeutic management of patients. Furthermore, it is worth noting that we did not simulate hot spots, mainly resulting from the accumulation of aerosols in the bronchi, that can make this method based on the intensity of the maximal pixel value totally inaccurate.

The Z-score-based threshold method (ZTh) was conceptually attractive. Indeed, physiological interindividual variabilities of regional lung function is a challenge for automatic delineation of PVOI. Integrating the standard deviation in a pixel by pixel analysis was therefore promising. However, spatial correlation was lower as compared with the 2 others methods, with a mean DICE $E_{P E}$ of 0.67 , showing that the method is inaccurate to delineate PE. There was a large overestimation of PVOI for small PE, with up to $250 \%$ of relative difference, and a systemic under estimation for medium and large PE (around - 50\% of relative difference). The over estimation of small PVOI was also due to the delineation of non PE areas, mainly in the center of lungs, as shown in Fig. 8. On the other hand, there was an underestimation of PE volumes in the periphery of the lungs, as shown in Fig. 9. The explanation is provided by the analysis of the normal parametric maps, which show low uptake on mean map and high variability on standard SD maps on the peripheral regions of the lungs (see Fig. 1). This was likely due to free-form registration during the NSDmap creation process, as there is a high variability in patients' size, and thus in lungs deformation, resulting in the non delineation of PE areas in the periphery of the lungs. Conversely, this issue in the periphery of the lungs led to the choice of a very selective Z-score threshold, which provided the best mean $\mathrm{DICE}_{\mathrm{PE} \text { index }}$, but which resulted in the delineation of non PE areas in the center of lungs.

The relative difference threshold method showed the best results, in particular regarding the spatial correlation and the PVOI measurment for small and medium PE. Mean DICE $\mathrm{PE}$ coefficient was the highest $(0.85(0.08))$, regardless of the intensity of the anterior to posterior gradient (mean $\operatorname{DICE}_{\mathrm{PE}}=0.87(0.08), 0.87(0.06)$, and $0.86(0.06)$ with the weak, regular, and strong gradient) and the lung volume (mean $\mathrm{DICE}_{\mathrm{PE}}=0.81$ (0.11), 0.87 (0.06), and 0.81 (0.11) with the small, medium, and large lung volumes). However, the PVOI correlation analysis shows a measurement bias, which was also found in the Bland and Altman analysis (relative bias of 12\%), but with the smallest confidence interval of the 3 segmentation methods (- 40 to 16\%). As discussed above, a key result in a clinical perspective was that PVOI estimation was highly accurate for small and medium size PE. For large PE, there was a trend toward a small underestimation of PVOI, as shown in Fig. 9 . However, the clinical consequences are likely negligible. A likely explanation for this weak underestimation is the non delineation on the edge of the lungs, resulting from the lower spatial resolution of the normal mean map. As mentioned before, hotspots can affect deliniation methods. This method being based 
on mean intensity value normalization, it should be less affected. Nevertheless, it has to be clinically demonstrated.

There is only few data in the litterature about lung V/Q SPECT delineation in the context of PE. Derlin et al. [21] and Seiffert et al. [24] tested several maximum intensity thresholds and compared them to manual delineation of functional lungs. The best threshold was much higher as compared with our study (40\% vs 15\%) for Derlin, but closer for Seiffert [24] (18\% for left lung and $21 \%$ for right lung), illustrating the lake of robustness of the method, especially if there are hot spots. Wang et al. [25-27] proposed three segmentation methods (one threshold based method and two adaptive contouring methods), tested on Monte-Carlo simulations of homogeneous distributions of radioactivity within the lungs. The results, expressed as the ratio of the intersection and the union of the ground truth and the segmented volume, were variable depending on the count rates. The results were up to $97 \pm 2$ for high count rate, but ranged from $78 \% \pm 23$ to $90 \% \pm 9$ for low count rates, although the measurement was performed on the functioning lungs (and not on PE) and on simulated data without the intensity gradient. Cheimariotis et al. [28] proposed an active shape segmentation method and compared it with manual delineation. Although DICE indices were calculated on the functional lungs (i.e., not on PE volumes), which inherently improve the correlation of segmented lung volumes, the Dice coefficients were lower than in our study (DICE = 0.82 and 0.83 for left and right lungs respectively).

Our study has some limitations. First, we used a static plantom and we not simulated respiratory motion. However, based on the analysis of 73 normal coregistrated cases database [14], the impact of respiratory motion on the uptake variability in the basis areas was very low, especially as compared with the anterior to posterior gradient. Second, we only focused the analysis on perfusion images while the hallmark of acute PE with V/Q scintigraphy is the mismatched perfusion defect, i.e., areas with absent perfusion but preserved ventilation. There are many pulmonary conditions, such as chronic obstructive pulmonary disease or pneumonia, which can be the source of matched defects present on both perfusion and ventilation. On the other hand, the Meyer score [11] as long as recent studies that demonstrated the predictive value of the PVOI on PE recurrence $[9,10]$ only used perfusion images for PVOI quantification. Third, the DICE coefficients used in the calibration process, calculated on PE volumes, were relatively low and showed a large range, especially for the MaxTh and ZTh methods, which may question the reliability of the selected threshold and limit its generalizability. Of note however is that the DICE coefficients were much higher for the relative difference method. Fourth,while Monte Carlo simulation offers many advantages, including the reliability of the reference standard and the possibility to assess multiple methods and clinical scenario, it remains a phantom study. In particular, perfusion defects were artificially delineated and would not account for real patient variability. Thus, it is not possible to extrapolate our results to real V/Q SPECT/CT scans. Clinical studies are now warranted to assess whether this segmentation method can be used in daily practice to compute the PVOI.

\section{Conclusion}

In this phantom study, a delineation method based on a relative difference parametric map provided a good estimation of the PVOI, regardless of the extent of $\mathrm{PE}$, the 
intensity of the anterior to posterior gradient, and the whole lung volumes. As compared with manual segmentation method or segment counting method, this automated delineation method may provide a fast, accurate, and reproducible PVOI quantification. Clinical studies are now required to assess whether this segmentation method can be used in daily practice to compute the PVOI.

\begin{abstract}
Abbreviations
PE: Pulmonary embolism; V/Q: Ventilation/perfusion scan; SPECT: Single photon emission computed tomography; CT: Computed tomography; PVOI: Pulmonary vascular obstruction index; MaxTh: Relative to maximum intensity threshold method; ZTh: Z-score threshold method; RelDiffTh: Relative difference threshold method; CTEPH: Chronic thromboebolic pulmonary hypertension; SPM: Statistical parametric mapping; MELP: Medium energy low penetration; WLV $V_{P H}:$ Phantom's whole lungs volume; WLv: Simulation's whole lungs volume; FLV $V_{P H}:$ Phantom's functional lungs volume; FLvs: Simulation's functional lungs volume; PEV $\mathrm{PH}_{\mathrm{PH}}$ : Phantom's pulmonary embolism volume; PEvs: Simulation's pulmonary embolism volume; PVOl $\mathrm{PH}_{\text {: }}$ Phantom's pulmonary vascular obtruction index; $\mathrm{PVOl}_{\mathrm{s}}$ : Simulation's pulmonary vascular obtruction index; DICEPE: DICE index between PEVPH and PEv
\end{abstract}

\title{
Supplementary Information
}

The online version contains supplementary material available at https://doi.org/10.1186/s40658-021-00396-1.

Additional file 1.

Acknowledgements

Not applicable

\section{Authors' contributions}

$D B, P Y L R$, and PYS contributed to the designing of the study. DB, PYLR, PYS, JR, LW, PR, and RLP contributed to managing the imaging procedures. DB, PYLR, PYS, JR, LW, and CT contributed to the analysis of the data. All authors contributed to the writing of the manuscript. All authors read and approved the final manuscript.

\section{Funding}

None

Availability of data and materials

The datasets used and analyzed during the current study are available from the corresponding author on reasonable request.

\section{Declarations}

\section{Ethics approval and consent to participate}

All procedures performed in studies involving human participants were in accordance with the ethical standards of the institutional committee and with the 1964 Helsinki declaration and its later amendments. This study was approved by the local ethics committee B2019CE29 with the study number 29BRC19.0129. Informed consent was obtained from all individual participants included in the study.

\section{Consent for publication}

Informed consent was obtained from all individual participants included in the study

\section{Competing interests}

No conflicts of interest

\section{Author details}

${ }^{1}$ Service de Médecine Nucléaire, Centre Hospitalier Régional Universitaire de Brest, Brest, France. ²EA3878 GETBO, Université de Bretagne Occidentale, Brest, France. ${ }^{3}$ Service de Pneumologie, Centre Hospitalier Régional Universitaire de Brest, Brest, France.

Received: 19 March 2021 Accepted: 21 June 2021

Published online: 05 July 2021

\section{References}

1. Task FM, et al. 2019 ESC Guidelines for the diagnosis and management of acute pulmonary embolism developed in collaboration with the European Respiratory Society (ERS): The Task Force for the diagnosis and management of acute pulmonary embolism of the European Society of Cardiology (ESC). Eur Respir J. 2019;54(3):1901647.

2. Investigators, P. Value of the ventilation/perfusion scan in acute pulmonary embolism. Results of the prospective investigation of pulmonary embolism diagnosis (PIOPED). Jama. 1990;263(20):2753.

3. Wells PS, Ginsberg JS, Anderson DR, Kearon C, Gent M, Turpie AG, et al. Use of a clinical model for safe management of patients with suspected pulmonary embolism. Ann Intern Med. 1998;129(12):997-1005. https://doi.org/10.7326/0003-481 9-129-12-199812150-00002. 
4. Salaun P-Y, Couturaud F, le Duc-Pennec A, Lacut K, le Roux PY, Guillo P, et al. Noninvasive diagnosis of pulmonary embolism. Chest. 2011;139(6):1294-8. https://doi.org/10.1378/chest.10-1209.

5. Anderson DR, Kahn SR, Rodger MA, Kovacs MJ, Morris T, Hirsch A, et al. Computed tomographic pulmonary angiography vs ventilation-perfusion lung scanning in patients with suspected pulmonary embolism: a randomized controlled trial. Jama. 2007;298(23):2743-53. https://doi.org/10.1001/jama.298.23.2743.

6. Le Roux P-Y, et al. Safety of ventilation/perfusion single photon emission computed tomography for pulmonary embolism diagnosis. Eur J Nucl Med Mol Imaging. 2014;41(10):1957-64. https://doi.org/10.1007/s00259-014-2763-1.

7. Le Roux P-Y, et al. Additional value of combining low-dose computed tomography to V/Q SPECT on a hybrid SPECT-CT camera for pulmonary embolism diagnosis. Nucl Med Commun. 2015;36(9):922-30. https://doi.org/10.1097/MNM. 0000000000000351

8. Le Roux P-Y, et al. Pulmonary scintigraphy for the diagnosis of acute pulmonary embolism: a survey of current practices in Australia, Canada, and France. J Nucl Med. 2015;56(8):1212-7. https://doi.org/10.2967/jnumed.115.157743.

9. Tromeur C, Sanchez O, Presles E, Pernod G, Bertoletti L, Jego P, et al. Risk factors for recurrent venous thromboembolism after unprovoked pulmonary embolism: the PADIS-PE randomised trial. Eur Respir J. 2018;51(1): 1701202. https://doi.org/10.1183/13993003.01202-2017.

10. Pesavento R, et al. Impact of residual pulmonary obstruction on the long-term outcome of patients with pulmonary embolism. Eur Respir J. 2017:49(5):1601980

11. Meyer G, Collignon MA, Guinet F, Jeffrey AA, Barritault L, Sors H. Comparison of perfusion lung scanning and angiography in the estimation of vascular obstruction in acute pulmonary embolism. Eur J Nucl Med. 1990;17(6-8):3159. https://doi.org/10.1007/BF01268022.

12. Le Roux P-Y, Robin P, Salaun P-Y. New developments and future challenges of nuclear medicine and molecular imaging for pulmonary embolism. Thromb Res. 2018;163:236-41. https://doi.org/10.1016/j.thromres.2017.06.031.

13. Friston KJ. Statistical parametric mapping. In: Neuroscience databases: Springer; 2003. p. 237-50.

14. Bourhis D, Robin P, Essayan M, Abgral R, Querellou S, Tromeur C, et al. V/Q SPECT for the assessment of regional lung function: generation of normal mean and standard deviation 3-D Maps. Front Med. 2020;7. https://doi.org/10.3389/ fmed.2020.00143.

15. Bourhis D, Wagner L, Essayan M, Robin P, le Pennec R, Salaun PY, et al. Normal dual isotope V/Q SPECT model for Monte-Carlo studies. Front Med. 2020;7. https://doi.org/10.3389/fmed.2020.00461.

16. Ljungberg M, Strand S, King M. The SIMIND Monte Carlo program. Monte Carlo calculation in nuclear medicine: Applications in diagnostic imaging. Bristol: IOP Publishing; 1998. p. 145-63.

17. Toossi MB, et al. SIMIND Monte Carlo simulation of a single photon emission CT. J Med Phys Assoc Med Physicists India. 2010;35(1):42.

18. Ljungberg M. The SIMIND Monte Carlo Program Manual; 2017.

19. Zubal IG, Harrell CR, Smith EO, Rattner Z, Gindi G, Hoffer PB. Computerized three-dimensional segmented human anatomy. Med Phys. 1994;21(2):299-302. https://doi.org/10.1118/1.597290

20. Schneider CA, Rasband WS, Eliceiri KW. NIH Image to ImageJ: 25 years of image analysis. Nat Methods. 2012;9(7):671-5. https://doi.org/10.1038/nmeth.2089.

21. Derlin T, et al. Quantitation of perfused lung volume using hybrid SPECT/CT allows refining the assessment of lung perfusion and estimating disease extent in chronic thromboembolic pulmonary hypertension. Clin Nucl Med. 2018;43(6): e170-e177.

22. Bucknell NW, Hardcastle N, Bressel M, Hofman MS, Kron T, Ball D, et al. Functional lung imaging in radiation therapy for lung cancer: a systematic review and meta-analysis. Radiother Oncol. 2018;129(2):196-208. https://doi.org/10.1016/j.ra donc.2018.07.014.

23. Le Roux P-Y, et al. Automatic delineation of functional lung volumes with 68Ga-ventilation/perfusion PET/CT. EJNMMI Res. 2017;7(1):82. https://doi.org/10.1186/s13550-017-0332-x.

24. Seiffert AP, Gómez-Grande A, Pilkington P, Cara P, Bueno H, Estenoz J, et al. Automatic diagnosis of chronic thromboembolic pulmonary hypertension based on volumetric data from SPECT ventilation and perfusion images. Appl Sci. 2020;10(15):5360. https://doi.org/10.3390/app10155360.

25. Wang A, Yan H. Delineating low-count defective-contour SPECT lung scans for PE diagnosis using adaptive dual exponential thresholding and active contours. Int J Imaging Syst Technol. 2010;20(2):149-54. https://doi.org/10.1002/ ima.20222.

26. Wang A, Yan H. SPECT lung delineation via true 3D active contours. IAENG Int J Comput Sci. 2010;37(3):IJCS-37-3-01.

27. Wang A, Yan H. A new automated delineation method for SPECT lung scans using adaptive dual-exponential thresholding. Int J Imaging Syst Technol. 2007;17(1):22-7. https://doi.org/10.1002/ima.20094.

28. Cheimariotis $\mathrm{GA}$, al-Mashat $\mathrm{M}$, Haris $\mathrm{K}$, Aletras AH, Jögi J, Bajc M, et al. Automatic lung segmentation in functional SPECT images using active shape models trained on reference lung shapes from CT. Ann Nucl Med. 2018;32(2):94-104. https:// doi.org/10.1007/s12149-017-1223-y.

\section{Publisher's Note}

Springer Nature remains neutral with regard to jurisdictional claims in published maps and institutional affiliations. 